\title{
Use of photoelectron energy spectrum transfer equation for the measurement of a narrowband XUV pulse
}

\author{
GE YuCheng* \& HE HaiPing \\ School of Physics and State Key Laboratory of Nuclear Physics and Technology, Peking University, Beijing 100871, China
}

Received August 3, 2011; accepted September 21, 2011

\begin{abstract}
To study the time evolution of a molecular state in an ultra-fast chemical reaction, the use of shorter pulses with higher photon energy and narrower bandwidth for both pump and probe is necessary. However, quick and precise measurement of their detailed time structures is a challenge. Over the last decade, great efforts have been made to measure an attosecond extreme ultraviolet (XUV) pulse. To date, several methods have been developed to measure the pulse duration and completely reconstruct it. The attosecond spectral phase interferometry for direct electric field reconstruction (SPIDER) and attosecond frequency-resolved optical gating (FROG) techniques are often used. However, these methods use state-of-the-art experimental set-ups and complicated data analysis procedures. To develop attosecond metrology for practical use (e.g. timing, measurement, evaluation, calibration, optimization, pumping, probing), we propose a quick and analytical method to precisely observe an attosecond XUV pulse with laser-assisted photo-ionization. The method is based on determining the laser-related phase of each streaked electron and using a transfer equation for one-step pulse reconstruction without any time-resolved measurements, iterative calculations, or data fitting procedures. Temporal errors of the pulse reconstruction are calculated from the XUV bandwidth. Because the transfer equation establishes a direct connection between the XUV pulse properties, the crucial laser parameters (peak intensity, phase, carrier envelope phase), the atomic ionization potential, and the measured photoelectron energy spectrum, we can use it to study any one of these properties from other known information and probe the dynamic processes of an ultra-fast reaction.
\end{abstract}

attosecond measurement, photoelectron energy spectrum, laser phase determination method, transfer equation

Citation: Ge Y C, He H P. Use of photoelectron energy spectrum transfer equation for the measurement of a narrowband XUV pulse. Chin Sci Bull, 2012, 57: 843-848, doi: $10.1007 / \mathrm{s} 11434-011-4943-8$

Over the last decade, production and measurement of the duration of an attosecond ( 1 as $=10^{-18} \mathrm{~s}$ ) extreme ultra-violet (XUV) pulse has attracted increasing interest [1-6]. Highorder harmonic generation (HHG) has been a successful way to produce attosecond XUV pulses [7-9]. To characterize the time evolution of a molecular state in an ultra-fast chemical reaction, shorter pulses with higher photon energies and narrower bandwidths have been generated and used, but quickly and precisely measuring their detailed time structures for use remains a challenge. This can currently be attributed to the difficulty of attosecond measurements and the associated errors. Recently, different techniques such as phase-matching and spatial filtering have been used to analyze, produce and

*Corresponding author (email: gyc@pku.edu.cn) select an isolated attosecond pulse [10-12]. The latest measurement of an attosecond XUV pulse duration is 80 as [13]. However, researchers continue to improve the production of attosecond pulses and seek new methods to measure them for use. To date, several methods have been developed to measure the pulse duration and completely reconstruct it. For example, the temporal characteristics of a train of attosecond pulses were directly determined by measuring second-order autocorrelation traces [14]. Similar methods have been used for attosecond spectral phase interferometry for direct electric field reconstruction (SPIDER) and attosecond frequencyresolved optical gating (FROG) techniques [15-19], and for their extensions [20-22]. The cross-correlation technique has been widely used in characterizing pulse durations [15]. Laser-assisted photo-ionization asymmetry has been used 
to determine the pulse duration of an attosecond XUV pulse [23,24]; however, most measurements have been based on the "streak camera" principle [25-30]. The quantum theory of streaking measurement was recently constructed for complete pulse reconstruction that excluded any guesswork, but in principle, a sufficiently large set of measurements and a great amount of calculation have been necessary [31-33].

With the development of state-of-the-art laser systems and cross correlation technology for attosecond XUV pulses, both the laser carrier envelope phase (CEP) and the pulse temporal location can be stabilized with high precision. This motivated us to establish a quick and analytical way to observe an isolated narrowband attosecond XUV pulse by determining the related laser phase of each measured photoelectron. Because the laser-assisted photo-ionization refers to a quantum process of an atom in strong fields, the photoelectron laser phase determination method is based on calculation at the singleatom level using the strong field approximation (SFA) [34].

In this paper, we present transfer equations and use the photoelectron laser phase determination method to quickly reconstruct the detailed time structures of narrowband XUV pulses with different energies and time properties from quantum-mechanically calculated photoelectron energy spectra (PESs) and experimental data. The theoretical errors of the pulse reconstruction are quantitatively analyzed therein. The major difference between the proposed method and the FROG CRAB [20] is that we reconstruct an attosecond XUV pulse from only one measured PES, rather than from $\boldsymbol{a}$ large set of time-resolved PESs, as used in FROG for selfconsistency in the data. Thus, the efficiency and speed of PES measurement and pulse reconstruction can be greatly improved. The method provides an alternative way to tackle the problems that an attosecond measurement inevitably involves, which are currently because of technical limitations, such as pulse jitters in time and space, parameter instabilities (e.g. walks over a long experimental course), data fluctuations, etc. Because the transfer equation includes all of the parameters of the incident laser and the XUV pulse, and a PES measured with a single-shot (multi-shot detection) records the instantaneous (average) values of these parameters, the transfer equation can be used to analyze the factors affecting the results of the measurement.

\section{Laser-assisted photo-ionization}

For cross-correlation between the XUV pulse and a fewcycle streaking field, the PES can be calculated by applying SFA [34] from the probability amplitude for transitioning from the ground state $|0\rangle$ to a final momentum $(\boldsymbol{p})$ state $|\boldsymbol{p}\rangle$, $b(\boldsymbol{p})=i \int_{-\infty}^{\infty} \mathrm{d} t E(t) d_{x}\left[\boldsymbol{p}-\boldsymbol{A}_{L}(t)\right] \mathrm{e}^{-i \widetilde{S}(t)}$, where $\widetilde{S}(t)$ is the quasiclassical action, $\widetilde{S}(t)=\int_{t}^{\infty} \mathrm{d} t^{\prime}\left(\left[\boldsymbol{p}-\boldsymbol{A}_{L}\left(t^{\prime}\right)\right]^{2} / 2+I_{p}\right)$. Here $E(t)$ is the amplitude of the combined fields of the linearlypolarized laser $\left[\boldsymbol{E}_{L}(t)\right]$ and XUV $\left[\boldsymbol{E}_{X}(t)\right] . t$ denotes the photoelectron birth time, $\boldsymbol{E}_{L}(t)=E_{L}(t) \cos \left(\omega_{L} t+\Phi\right)(x$ direction $)$ and $\boldsymbol{A}_{L}(t)$ represent the laser electric field and its vector potential, respectively. $\Phi$ is the CEP and $I_{p}$ is the atomic ionization potential. $\boldsymbol{d}(\boldsymbol{p})=\langle\boldsymbol{p}|\boldsymbol{x}| 0\rangle$ denotes the atomic dipole matrix element for the bound-free transition, and $d_{x}(\boldsymbol{p})$ is the component parallel to the polarization axis.

We use a Gaussian-like time function, $F(t)=$ $\exp \left[-4 \ln 2(t)^{2} / \tau_{L}^{2}\right]$, with a full-width at half-maximum (FWHM) pulse duration, e.g. $\tau_{L}=7 \mathrm{fs}$, and the wavelength $\lambda_{L}=750 \mathrm{~nm}$, to describe the envelope of a laser electric field, shown as Figure 1(a). In calculations or experiments, a PES, $n(W)$, can be defined as the number of photoelectrons per unit energy. Practically, $n(W)=N_{e}(W) / E_{\text {bin }}$, where $N_{e}(W)$ is the number of photoelectrons collected at energy $W$ within a small size of energy bin, $E_{\text {bin }}$, e.g. 1.0, $0.5 \mathrm{eV}$, or less. $\tau_{X}$ is defined as the duration of the XUV pulse (FWHM). The XUV angular frequency, $\omega_{X}$, is assumed to be a constant equal to the center frequency of the narrowband pulse. Using these parameters, Figure 1(b) plots PESs (solid lines), $n(W)$, emitted from hydrogen atoms $\left(I_{p}=13.6 \mathrm{eV}\right)$, calculated with different XUV energies, $\hbar \omega_{X}=(90.0,283.7) \mathrm{eV}$, and laser (peak) intensity, $S=$ $4 \times 10^{13} \mathrm{~W} / \mathrm{cm}^{2}, \Phi=0^{\circ}$, and $\theta=0^{\circ}(\theta$ is the observation
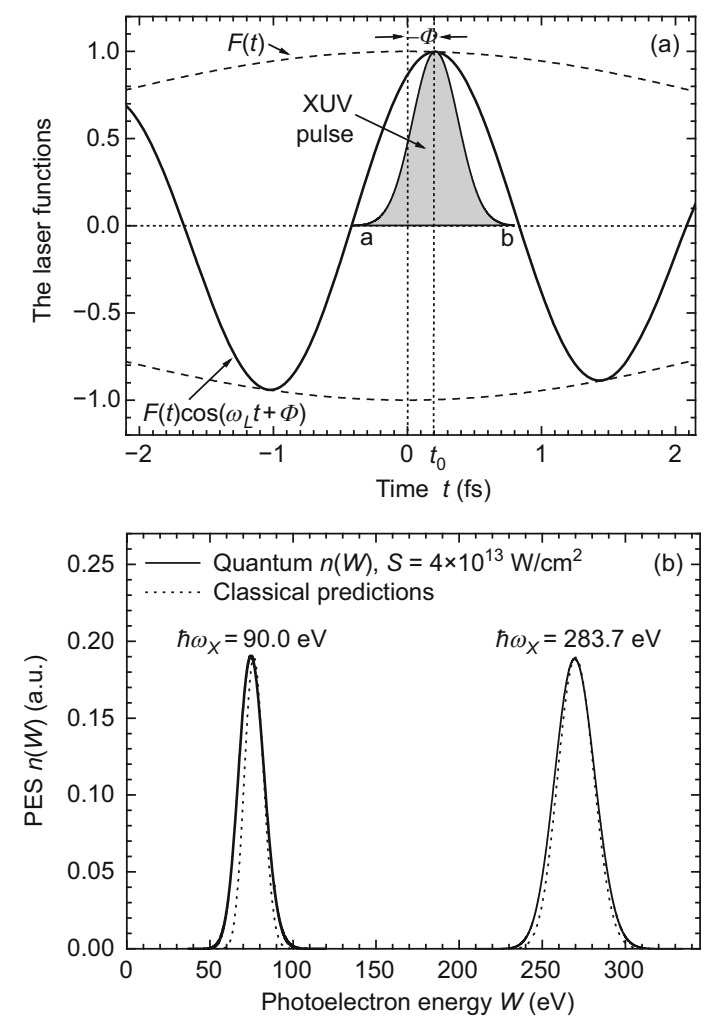

Figure 1 (a) Illustration of cross-correlation between a few-cycle dressing laser and an attosecond XUV pulse. Dashed and solid lines depict the time functions of the laser envelope $F(t)$ and the laser electric field $F(t) \cos \left(\omega_{L} t+\right.$ $\Phi$ ) (with amplitude $1, \Phi=-\omega t_{0}$ ), respectively. The shaded Gaussian-like profile is an XUV pulse being cross-correlated to half of a laser optical cycle (phases $-\pi / 2-\pi / 2$ ). (b) Solid lines: PESs quantum-mechanically calculated with two different photon energies for monochromatic XUV pulses $\left[\hbar \omega_{X}=(90.0,283.7) \mathrm{eV}\right]$ in the presence of an intense $7 \mathrm{fs} 750 \mathrm{~nm}$ dressing laser $\left(S=4 \times 10^{13} \mathrm{~W} / \mathrm{cm}^{2}, U_{p}=2.1 \mathrm{eV}\right)$. Dotted lines: classical predictions of PESs. 
angle between $\boldsymbol{p}$ and the direction of laser polarization). The XUV pulses of $\tau_{X}=0.150 \mathrm{fs}$ are temporally located at $t=0$. The classical predictions of each $n(W)$ (dotted lines in Figure 1(b)) calculated using the saddle point method [28,34] are also shown for comparison only. The small differences between the calculated PESs and their classical predictions have directed us to derive the transfer equations for one-step pulse reconstruction.

\section{Transfer equations for pulse reconstruction}

From the semi-classical point of view [1], the value of the final velocity $v(t)$ of a photoelectron born at time $t$ and moving at $\theta=0^{\circ}$ can be written as $v(t)=v_{i}(t)+$ $2 \sqrt{U_{p} / m_{e}} F(t) \sin \left(\omega_{L} t+\Phi\right)$, where $U_{p}$ is the laser ponderomotive potential $(\propto S), m_{e}$ is the electron mass, $v_{i}(t)=$ $\sqrt{2 W_{i}(t) / m_{e}} \approx \sqrt{2 W_{0} / m_{e}}=\sqrt{2\left(\hbar \omega_{X}-I_{p}\right) / m_{e}}$ is the initial photoelectron velocity, and $W_{i}(t)$ is the corresponding initial photoelectron energy, centered at $W_{0} . W_{0^{\circ}}(t)=m_{e} v^{2}(t) / 2$ depicts the final energy of a photoelectron born at $t$ and observed at $\theta=0^{\circ}$ :

$$
\begin{aligned}
W_{0^{\circ}}(t)= & W_{0}+2 U_{P} F^{2}(t) \sin ^{2}\left(\omega_{L} t+\Phi\right) \\
& +\sqrt{8 U_{P} W_{0}} F(t) \sin \left(\omega_{L} t+\Phi\right) .
\end{aligned}
$$

On the other hand, the equation of $v(t)$ (in the line above) can be written equivalently as

$$
\sin \left(\omega_{L} t+\Phi\right)=\frac{\sqrt{W_{0^{\circ}}(t)}-\sqrt{W_{0}}}{\sqrt{2 U_{P}} F(t)} .
$$

We use this equation to determine the photoelectron laser phase from the measured photoelectron energy $W$, instead of $W_{0} \circ(t)$.

High laser intensity is usually used to populate photoelectrons over a broad energy range; however, both the background and the laser-induced part of the above-threshold ionization (ATI) spectrum limit the laser intensity $[28,35]$. According to the simple condition [28], $8 U_{P} \leqslant \hbar \omega_{X}-I_{p}$, a moderate intensity of $S=1.2 \times 10^{14} \mathrm{~W} / \mathrm{cm}^{2}\left(U_{P}=6.3 \mathrm{eV}\right.$ for a $750 \mathrm{~nm}$ laser) or lower ensures that the PESs are fully separated from the ATI spectra for the XUV frequencies concerned.

Figure 2 illustrates the derivation of the transfer equation for pulse reconstruction with $\theta=0^{\circ}$ measurement for a narrowband attosecond XUV pulse. The dashed-dotted line in Figure 2 depicts the final energy, $W_{0^{\circ}}(t)$, of a photoelectron born at a different time, $t$. The highlighted curve APB denotes one-half of the laser period, wherein the XUV pulse is temporally located. $f(t)$ is assumed to be the temporal profile of the XUV pulse. As depicted in Figure 2, the photoelectrons generated from time $t=a$ to $t=b$ will populate along the energy axis from $W 1=W_{0^{\circ}}(a)$ to $W 2=W_{0^{\circ}}(b)$. Using an excitation function, $\sigma\left(\hbar \omega_{X}\right)$, which is the photoionization cross section of the active gas as a function of

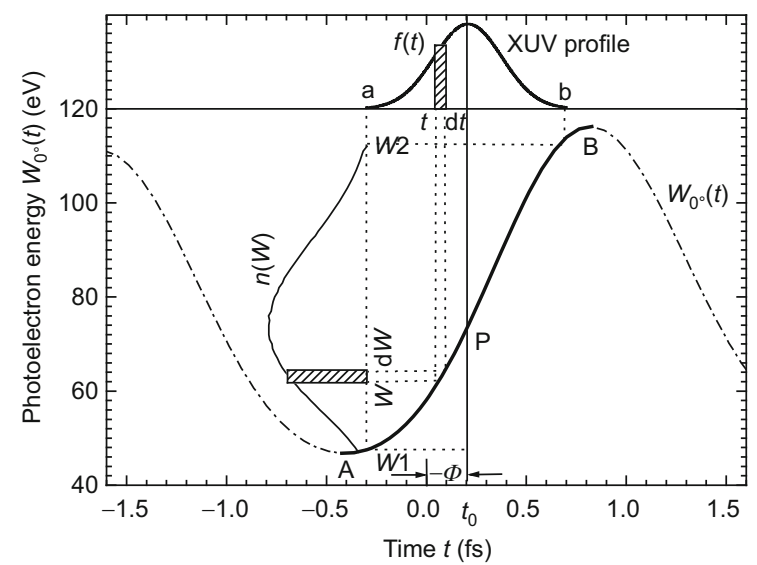

Figure 2 Derivation of the transfer equation for pulse reconstruction of a narrowband attosecond XUV pulse.

the XUV photon energy $\hbar \omega_{X}$, the relationship between the pulse intensity and the measured PES can be established. The number of photoelectrons collected at energy $W$ within a small interval $\mathrm{d} W$ is proportional to the number of XUV photons at time $t$ within a small interval $\mathrm{d} t$, i.e. $n(W) \mathrm{d} W=$ $g \eta \rho \sigma\left(\hbar \omega_{X}\right) f(t) \mathrm{d} t$, where $g$ is a constant geometric factor of the spectrometer, $\eta$ is the detection efficiency, and $\rho$ is the atomic density of the active gas. Because $\sigma\left(\hbar \omega_{X}\right)$ can be approximately set as a constant for a narrowband attosecond XUV pulse, we use another constant, $\mu$, instead of $1 / g \eta \rho \sigma\left(\hbar \omega_{X}\right)$. The intensity-related transfer equation then has the following simple form:

$$
f(t)=\frac{\mathrm{d} W_{0^{\circ}}(t)}{\mathrm{d} t} \cdot \mu n(W) .
$$

Here, we use $\mathrm{d} W_{0^{\circ}}(t) / \mathrm{d} t$ instead of $\mathrm{d} W / \mathrm{d} t$ for $\theta=0^{\circ}$ measurements. $\mathrm{d} W_{0^{\circ}}(t) / \mathrm{d} t$ is a laser-related time-dependent factor, which can be theoretically calculated for the measurement. Eq. (3) is the transfer equation to be used in calculating the unknown temporal structure, $f(t)$, from a proportional term of the measured PES, $\mu n(W)$. Usually, $\mu$ is set to 1 for simplicity. In the case of a "cosine"-like field and $\theta=0^{\circ}$ measurement (or "cosine" like field but $\theta=180^{\circ}$ ), extra "." signs occur in front of the last terms on the right-hand sides of eqs. (1)-(3).

\section{Temporal errors due to XUV bandwidth}

Usually, the chirp of the XUV pulse is a time-dependent frequency profile $\omega_{X}(t)$. For a narrowband XUV pulse, we can use a time-dependent intensity profile $f(t)$ as a simplified description of the pulse for quick measurement. In the following, we derive a formula to calculate the temporal errors of the pulse reconstruction due to the XUV bandwidth.

Eq. (1) is a laser field modulation equation. Considering a small shift $\Delta W$ of the XUV photon energy, $\hbar \omega_{X}$, or the initial photoelectron energy, $W_{0}$, the final energy, $W_{0^{\circ}}$, of a photoelectron generated at time $t$ will have a shift $\Delta W_{0^{\circ}} . \Delta W$ and 
$\Delta W_{0^{\circ}}$ satisfy $W_{0^{\circ}}+\Delta W_{0^{\circ}}=\left(W_{0}+\Delta W\right)+2 U_{P} F^{2}(t) \sin ^{2}\left(\omega_{L} t+\right.$ $\Phi)+\sqrt{8 U_{P}\left(W_{0}+\Delta W\right)} F(t) \sin \left(\omega_{L} t+\Phi\right)$. In fact, we use another equation, $W_{0^{\circ}}+\Delta W_{0^{\circ}}=W_{0}+2 U_{P} F^{2}(t+\Delta t) \sin ^{2}\left[\omega_{L}(t+\right.$ $\Delta t)+\Phi]+\sqrt{8 U_{P} W_{0}} F(t+\Delta t) \sin \left[\omega_{L}(t+\Delta t)+\Phi\right]$, to determine the photoelectron birth time $t^{\prime}=t+\Delta t$, where $W_{0}$ is used as a constant and $\Delta t$ is a time shift of $t$. If (i) $\Delta W \ll W_{0}$, i.e. $W_{0}+\Delta W \approx W_{0}$; (ii) $F(t) \approx 1$ and $F(t+\Delta t) \approx 1$, i.e. both $t$ and $t+\Delta t$ are close to 0 ; and (iii) $2 U_{P} F^{2}(t+\Delta t) \sin ^{2}\left[\omega_{L}(t+\Delta t)+\Phi\right]-2 U_{P} F^{2}(t) \sin ^{2}\left(\omega_{L} t+\Phi\right)$ can be neglected in comparison with the other terms, we can then solve $\Delta W$ from

$$
\begin{aligned}
\Delta W & \approx \omega_{L} \sqrt{8 U_{P} W_{0}} F(t) \cos \left(\omega_{L} t+\Phi\right) \cdot \Delta t \\
& \approx \frac{\mathrm{d} W_{0^{\circ}}(t)}{\mathrm{d} t} \cdot \Delta t .
\end{aligned}
$$

For an attosecond XUV pulse with a narrow bandwidth $\Delta \hbar \omega_{X} \equiv \Delta W_{B W} \ll W_{0}$, we use $\Delta W_{B W}$ instead of $\Delta W$ in eq. (4). Therefore, an energy bandwidth $\Delta W_{B W}$ of the XUV pulse will result in a temporal error $\Delta t$ for the determined photoelectron birth time. The instantaneous temporal error $\Delta t$ of the calculated $f(t)$ can be expressed as

$$
\Delta t=\frac{\Delta W_{B W}}{\left|\mathrm{~d} W_{0^{\circ}}(t) / \mathrm{d} t\right|} .
$$

From eq. (5), we see that the temporal errors at different times in the pulse reconstruction depend on the following factors: XUV photon energy $\hbar \omega_{X}$, XUV bandwidth $\Delta W_{B W}$, laser frequency $\omega_{L}$, laser intensity $S$, and laser phase $\omega_{L} t+\Phi$. For example, temporal errors decrease linearly with XUV bandwidth. Theoretically, we can use another three factors, $\alpha$ (root-mean-square intensity difference), $\beta$ (mean time difference), and $\varepsilon$ (root-mean-square time difference) to evaluate the results of a pulse reconstruction for an attosecond monochromatic XUV pulse. They are defined as follows:

$$
\begin{aligned}
& \alpha=\sqrt{\frac{1}{N} \sum_{n=1}^{N}\left[f\left(t_{n}\right)-f_{0}\left(t_{n}\right)\right]^{2},} \\
& \beta=\frac{1}{N} \sum_{n=1}^{N} \Delta t_{n}, \\
& \gamma=\sqrt{\frac{1}{N} \sum_{n=1}^{N}\left(\Delta t_{n}\right)^{2} .}
\end{aligned}
$$

Here, $f_{0}\left(t_{n}\right)$ is the value of the initial XUV pulse intensity profile $f_{0}(t)$ at time $t=t_{n} . t_{n}, \Delta t_{n}$, and $f\left(t_{n}\right)$ are values of the photoelectron birth time, the time difference between $f_{0}(t)$ and $f\left(t_{n}\right)\left[f_{0}(t)=f\left(t_{n}\right)\right]$, and the intensity retrieved from the photoelectron energy $W=W_{n}(n=1,2, \cdots, N)$ of the calculated PES, respectively. The values of $\alpha, \beta$, and $\gamma$ characterize the quality of the pulse reconstruction and the precision of the photoelectron laser phase determination. Obviously, the smaller the values of $\alpha, \beta$, and $\gamma$, the better the pulse reconstructions.

\section{Pulse reconstructions with calculated PESs}

To illustrate single-pulse reconstructions for monochromatic attosecond XUV pulses, we use the same laser as that used in the PES calculations of Figure 1(a) with $\Phi=0^{\circ}$. The dotted lines (overlapped by solid lines) in Figures 3(a) and (b) correspond to the initial XUV pulse profiles $f_{0}(t)$ with $\tau_{X}=0.4$
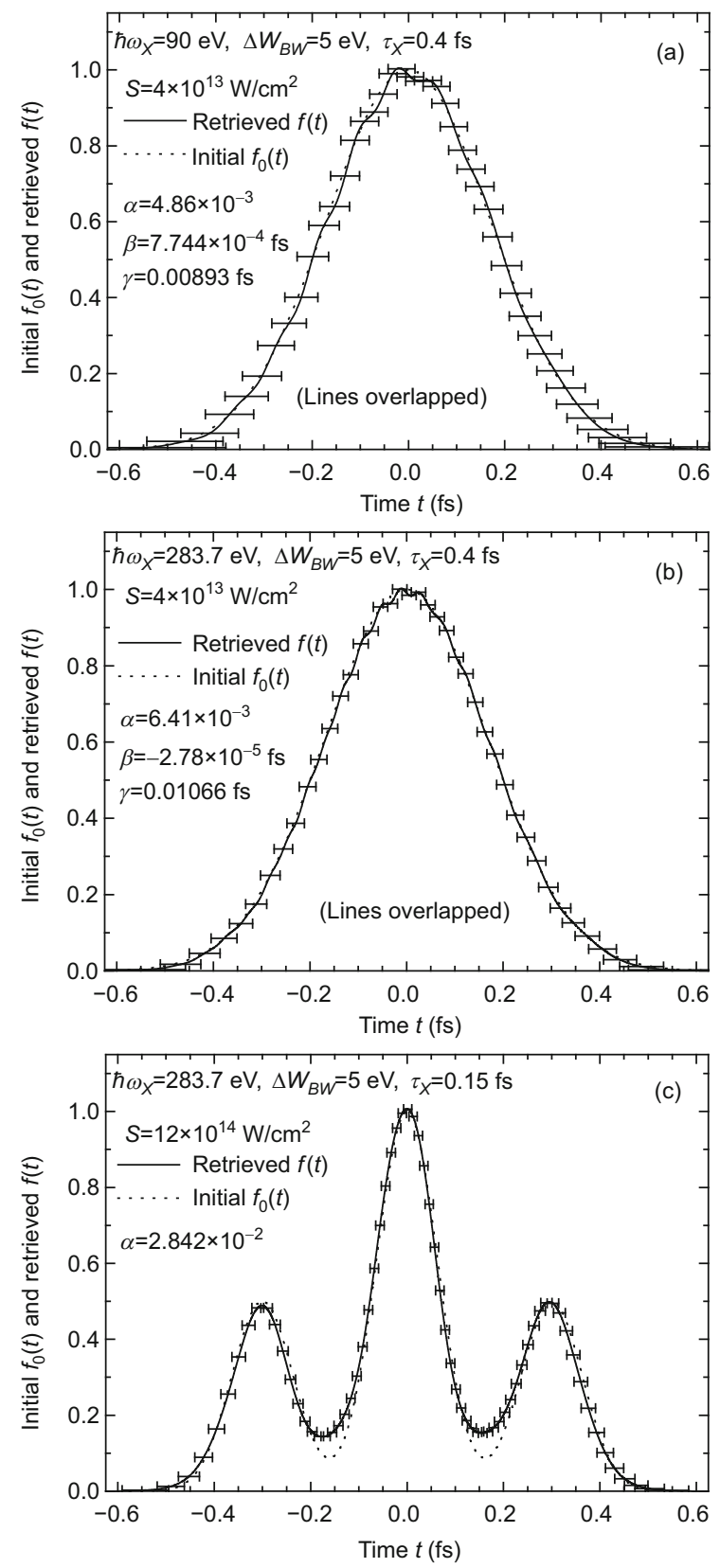

Figure 3 (a) and (b) Single-pulse reconstructions with $\tau_{X}=0.4 \mathrm{fs}, S=$ $4 \times 10^{13} \mathrm{~W} / \mathrm{cm}^{2}$, and $\hbar \omega_{X}=(90,283.7) \mathrm{eV}$, respectively. Dotted lines: the initial intensity profiles of the XUV pulses. Solid lines: pulse profiles reconstructed from quantum-mechanically calculated PESs. The error-bar lengths denote the temporal errors of the pulse reconstructions at different times, calculated using $\Delta W_{B W}=5 \mathrm{eV}$. (c) Similar to (a) and (b), a multi-pulse reconstruction with $\tau_{X}=0.15 \mathrm{fs}, S=1.2 \times 10^{14} \mathrm{~W} / \mathrm{cm}^{2}, \hbar \omega_{X}=283.7 \mathrm{eV}$. 
fs, and $\hbar \omega_{X}=(90,283.7) \mathrm{eV}$, respectively. The laser intensity and the XUV bandwidth are $S=4 \times 10^{13} \mathrm{~W} / \mathrm{cm}^{2}$ and $\Delta W_{B W}=5 \mathrm{eV}$, respectively. The solid lines correspond to the intensity profiles reconstructed from the quantummechanically calculated PESs using the transfer eqs. (2) and (3). The error-bar lengths represent the temporal errors at different times, calculated using eq. (5). Note that the reconstructed pulse profiles are almost the same as the initial profiles (lines overlapped). The small differences at different times are a result of the quantum enhancements of the laser-assisted photo-ionizations. The small values of $\alpha=4.86 \times 10^{-3}, 6.41 \times 10^{-3} ; \beta=7.744 \times 10^{-4}$ fs, $2.78 \times 10^{-5}$ fs; and $\gamma=0.00893$ fs, 0.01066 fs (shown in Figures 3(a) and (b), respectively) indicate that the calculations using the transfer equations can produce successful pulse reconstructions. Similarly, Figure 3(c) shows a successful multi-pulse reconstruction of one higher pulse with two side pulses, each of $0.150 \mathrm{fs}$ at FWHM, separated by $0.300 \mathrm{fs}$, implying that the transfer equations can be used for complex temporal structure reconstruction.

\section{Pulse reconstructions with measured PESs}

Because of the lack of PES measured with narrowband XUV pulses, we can use streaked spectra from the attosecond transient recorder measurement of photoelectron emission from neon excited with a $93 \mathrm{eV}$ sub-femtosecond pulse [4] for quick pulse reconstruction. Figure 4(a) shows a PES measured in the absence of a laser field. The spectrum gives a Gaussian fit bandwidth for the isolated XUV pulses, $\Delta W_{B W}=$ $8.5 \mathrm{eV}$ (FWHM). Figure 4(b) shows the spectrum recorded with adjacent zero transitions of $A_{L}(t)$. Filled circles in Figure $4(\mathrm{c})$ show the pulse profile $f(t)$ reconstructed from the PES in Figure 4(b) with $S=1.7 \times 10^{13} \mathrm{~W} / \mathrm{cm}^{2}$. We can obtain a Gaussian fit profile centered at $t_{c}=0.038 \mathrm{fs}$ with a pulse duration (FWHM) $\Delta T=0.251 \mathrm{fs}$, which is in excellent agreement with the results reported in [4] (0.250 fs). Errorbar lengths at different times are calculated with $\Delta W_{B W}=8.5$ $\mathrm{eV}$, showing a minimum value of $\Delta t_{\min }=0.148 \mathrm{fs}$. In the experiments, a small bandwidth (e.g. $5 \mathrm{eV}$ and less) bandpass filter has been used to isolate an XUV pulse [1], so we re-calculate the error-bar lengths with an assumed value of $\Delta W_{B W}=3 \mathrm{eV}$, giving a minimum value of $\Delta t_{\min }=0.052 \mathrm{fs}$.

Despite the broad pulse bandwidth and the large data fluctuations, the results of the above pulse reconstruction have successfully yielded information about the pulse shape, duration and timing. If the pulse bandwidth can be decreased to a much smaller value, e.g. $\Delta W_{B W}<4 \mathrm{eV}$, the information from the reconstructed pulse can be used as reference values to analyze the status associated with pulse generation, isolation, transportation and detection. Increasing the stability of the laser parameters and decreasing the bandwidth of the isolated XUV pulse may greatly improve the efficiency and precision of attosecond pulse reconstruction and timing.
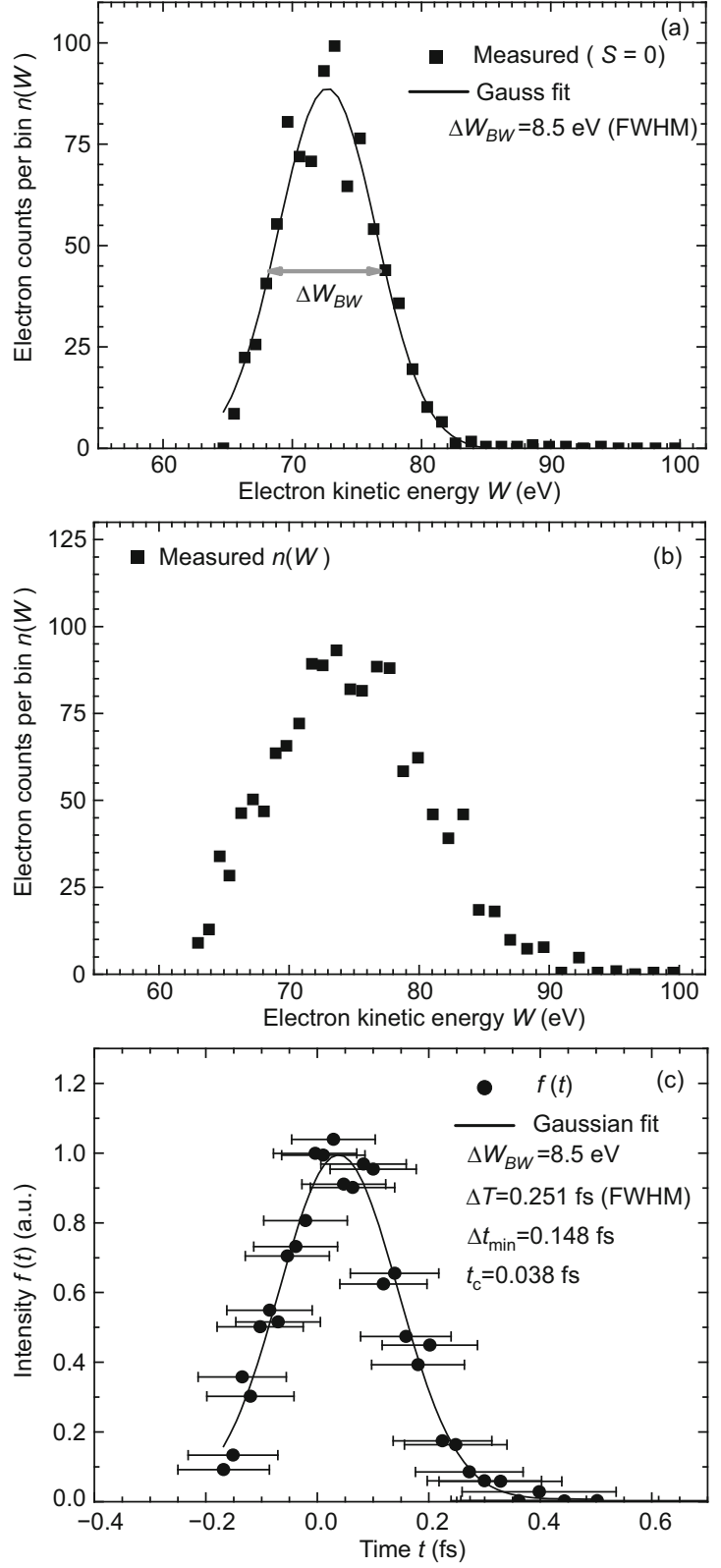

Figure 4 Use of one-step pulse reconstruction. (a) and (b) Spectra reprinted from [4]. (a) Spectrum without streaking. (b) Streaked spectrum. (c) Filled circles: reconstructed pulse. The error-bar lengths are calculated with $\Delta W_{B W}=8.5 \mathrm{eV}$.

\section{Conclusion}

A strong linearly-polarized few-cycle laser can be used as an attosecond ruler to precisely measure the detailed temporal structures of a narrowband attosecond XUV pulse by using the transfer equations presented here. The temporal errors generated in one-step pulse reconstruction because of the quantum effects are much smaller than those due to the bandwidth of the XUV pulse. The method presented here is capable of producing more time information for the XUV pulse, including possible asymmetry and side-peak structures. The following is a procedure that can be used to measure the PES 
and perform pulse reconstruction without any prior guesswork. (i) Measure the center energy, $\hbar \omega_{X}\left(W_{0}=\hbar \omega_{X}-I_{p}\right)$, and the energy bandwidth, $\Delta W_{B W}$, of the XUV pulse in the absence of the laser beam. (ii) Temporally locate the XUV pulse around $t=0$ (if $\Phi=0^{\circ}$ ) in a multi-shot experiment. (iii) Measure the PES $n(W)$ at $\theta=0^{\circ}$ (or $180^{\circ}$ ). (iv) Solve the photoelectron birth time, $t$, for each spectral position, $W$, from eq. (2), and calculate the differential term, $\mathrm{d} W_{0^{\circ}}(t) / \mathrm{d} t$, according to eq. (1). (v) Derive the unknown temporal structure, $f(t)$, of the XUV pulse using eq. (3). (vi) Calculate the temporal errors, $\Delta t$, at different times with the XUV bandwidth, $\Delta W_{B M}$, using eq. (5). In practical terms, the current result should be used as a reference to temporally locate the pulses for the next measurement.

Calculations demonstrate that the method of one-step pulse reconstruction can work for a broad range of XUV photon energies (from tens of $\mathrm{eV}$ to $\mathrm{keV}$ and higher), and has a temporal measurement range of one-half of the laser period. Because no iterative calculations or data analysis of great numbers of time-resolved PES measurements are needed, the method is advantageous for quick (online) pulse evaluation and measurement. Also, we can use the transfer equations to study a specified parameter and its time evolution, such as laser intensity, phase, CEP, or $I_{p}$, with measured PESs and other known information.

We are grateful to Prof. Ferenc Krausz and Reinhard Kienberger at MaxPlanck-Institut fuer Quantenoptik for discussion and the experimental data reprinted here. This work was supported by the National Natural Science Foundation of China (10827505 and 10675014).

1 Drescher M, Hentschel M, Kienberger R, et al. X-ray pulses approaching the attosecond frontier. Science, 2001, 291: 1923-1927

2 Hentschel M, Kienberger R, Spielmann Ch, et al. Attosecond metrology. Nature, 2001, 414: 509-513

3 Drescher M, Hentschel M, Kienberger R, et al. Time-resolved atomic inner-shell spectroscopy. Nature, 2002, 419: 803-807

4 Kienberger R, Goulielmakies E, Uiberacker M, et al. Atomic transient recorder. Nature, 2004, 427: 817-821

5 Goulielmakis E, Uiberacker M, Kienberger R, et al. Direct measurement of light waves. Science, 2004, 305: 1267-1269

6 Sansone G, Benedetti E, Calegari F, et al. Isolated single-cycle attosecond pulses. Science, 2006, 314: 443-446

7 Spielmann Ch, Burnett N H. Generation of coherent X-rays in the water window using 5-femtosecond laser pulses. Science, 1997, 278: 661664

8 Schnürer M, Spielmann Ch, Wobrauschek P, et al. Coherent 0.5-keV $\mathrm{X}$-ray emission from helium driven by a sub-10-fs laser. Phys Rev Lett, 1998, 80: 3236-3239

9 Kienberger R, Goulielmakis E, Uiberacker M, et al. Single sub-fs soft$\mathrm{X}$-ray pulses: Generation and measurement with the atomic transient recorder. J Mod Opt, 2005, 52: 261-275

10 Mairesse Y, Bohan A D, Frasinski L J, et al. Optimization of attosecond pulse generation. Phys Rev Lett, 2004, 93: 163901-163904

11 Gaarde M B, Schafer K J. Generating single attosecond pulses via spa- tial filtering. Opt Lett, 2006, 31: 3188-3190

12 Schiessl K, Ishikawa K L, Persson E, et al. Generating single attosecond pulses via spatial filtering. Phys Rev Lett, 2007, 99: 2539031-2939034

13 Goulielmakis E, Schultze M, Hofstetter M, et al. Single-cycle nonlinear optics. Science, 2008, 320: 1614-1617

14 Tzallas P, Charalambidis D, Papadogiannis N A, et al. Direct observation of attosecond light bunching. Nature, 2003, 426: 267-271

15 Quéré F, Itatani J, Yudin G L, et al. Attosecond spectral shearing interferometry. Phys Rev Lett, 2003, 90: 0739021-0739024

16 Kobayashi Y, Sekikawa T, Nabekawa Y, et al. 27-fs extreme ultraviolet pulse generation by high-order harmonics. Opt Lett, 1998, 23: 64-66

17 Sekikawa T, Ohno T, Yamazaki T, et al. Pulse compression of a highorder harmonic by compensating the atomic dipole phase. Phys Rev Lett, 1999, 83: 2564-2567

18 Norin J, Mauritsson J, Johansson A, et al. Time-frequency characterization of femtosecond extreme ultraviolet pulses. Phys Rev Lett, 2002 , 88: 193901-193904

19 Mauritsson J, Johnsson P, López-Martens R, et al. Measurement and control of the frequency chirp rate of high-order harmonic pulses. Phys Rev A, 2004, 70: 021801-021804(R)

20 Mairesse Y, Quéré F. Frequency-resolved optical gating for complete reconstruction of attosecond bursts. Phys Rev A, 2005, 71: 011401011404(R)

21 Quéré F, Mairesse Y, Itatani J. Temporal characterization of attosecond XUV fields. J Mod Opt, 2005, 52: 339-360

22 Mauritsson J, Johnsson P, López-Martens R, et al. Probing temporal aspects of high-order harmonic pulses via multi-colour, multi-photon ionization processes. J Phys B, 2005, 38: 2265-2278

23 Bandrauk A D, Chelkowski S, Shon N H. Measuring the electric field of few-cycle laser pulses by attosecond cross correlation. Phys Rev Lett, 2002, 89: 2839031-2839034

24 Bandrauk A D, Chelkowski S, Shon N H. How to measure the duration of subfemtosecond xuv laser pulses using asymmetric photoionization. Phys Rev A, 2003, 68: 041802-041805(R)

25 Constant E, Taranukhin V D, Stolow A, et al. Methods for the measurement of the duration of high-harmonic pulses. Phys Rev A, 1997, 56: 3870-3878

26 Scrinzi A, Geissler M, Brabec T. Attosecond cross correlation technique. Phys Rev Lett, 2001, 86: 412-415

27 Itatani J, Quéré F, Yudin G L, et al. Attosecond spectral shearing interferometry. Phys Rev Lett, 2002, 88: 1739031-1739034

28 Kitzler M, Milosevic N, Scrinzi A, et al. Quantum theory of attosecond XUV pulse measurement by laser dressed photoionization. Phys Rev Lett, 2002, 88: 1739041-1739044

29 Scrinzi A, Ivanov M Yu, Kienberger R, et al. Attosecond physics. J Phys B: At Mol Opt Phys, 2006, 39: R1-R37

30 Cavalieri A L, Müller N, Uphues Th, et al. Attosecond spectroscopy in condensed matter. Nature, 2007, 449: 1029-1032

31 Yakovlev V S, Bammer F, Scrinzi A. Attosecond streaking measurements. J Mod Opt, 2005, 52: 395-410

32 Kosik E M, Corner L, Wyatt A S, et al. Complete characterization of attosecond pulses. J Mod Opt, 2005, 52: 361-378

33 Nisoli M, Sansoni G. New frontiers in attosecond science. Prog Quant Electr, 2009, 33: 17-59

34 Lewenstein M, Balcou P, Ivanov M Y, et al. Theory of high-harmonic generation by low-frequency laser fields. Phys Rev A, 1994, 49: 2117 2132

35 Milosevic D B, Ehlotzky F. Coulomb and rescattering effects in abovethreshold ionization. Phys Rev A, 1998, 58: 3124-3125

Open Access This article is distributed under the terms of the Creative Commons Attribution License which permits any use, distribution, and reproduction in any medium, provided the original author(s) and source are credited. 